\title{
Time-domain full waveform inversion using the gradient preconditioning based on transmitted
} waves energy

\author{
Xiao-bo Zhang ${ }^{1}$, Jun Tan ${ }^{1}$, Peng Song ${ }^{* 1}$, Zhao-lun Liu' ${ }^{2}$, Jin-shan Li', Dong-ming Xia ${ }^{1}$ \\ ${ }^{1}$ Ocean University of China \& Qingdao National Laboratory for Marine Science and Technology \& Key Lab of \\ Submarine Geosciences and Prospecting Techniques Ministry of Education, ${ }^{2}$ King Abdullah University of \\ Science and Technology
}

\section{Summary}

The gradient preconditioning approach based on seismic wave energy can effectively avoid the huge storage consumption in the gradient preconditioning algorithms based on Hessian matrices in time-domain full waveform inversion (FWI), but the accuracy is affected by the energy of reflected waves when strong reflectors are present in velocity model. To address this problem, we propose a gradient preconditioning method, which scales the gradient based on the energy of the "approximated transmitted wavefield" simulated by the nonreflecting acoustic wave equation. The method does not require computing or storing the Hessian matrix or its inverse. Furthermore, it can effectively eliminate the effects caused by geometric diffusion and non-uniformity illumination on gradient. The results of model experiments confirm that the time-domain FWI using the gradient preconditioning based on transmitted waves energy can achieve higher inversion precision for high-velocity body and the deep strata below when compared with using the gradient preconditioning based on seismic waves energy.

\section{Introduction}

As the gradient of traditional FWI is affected by geometric diffusion and non-uniformity illumination, the velocity updates in middle-shallow strata always dominate the inversion process. Accordingly, the inversion convergence in deep strata and even the whole region slows down with low accuracy. Experts propose solutions to address this problem, including depth-weighted gradient method (Wang et al, 2009), and gradient preconditioning based on Hessian matrices, such as approximated Hessian matrix (Pratt et al, 1998, Shin et al, 2001), pseudo-Hessian matrix (Choi et al, 2008), and L-BFGS algorithm (Brossier et al, 2009).

A gradient preconditioning method based on seismic waves energy has been proposed by Zhang et al $(2011,2012)$ in order to avoid computing and storing large-scale matrix and eliminate the impact caused by geometric diffusion and non-uniformity illumination on gradient. The model experiment indicates that the inversion accuracy of this method is superior to the algorithm based on the pseudoHessian matrix (Zhang et al, 2012). For the gradient preconditioning method based on seismic wave energy, the computational accuracy of gradient preconditioning operator is seriously disturbed by the reflected wave information in seismic wavefield when strong reflectors exist in the velocity model. Therefore, we propose a gradient preconditioning method based on seismic transmitted wave energy, which simulates the "approximated transmitted wavefield" based on the nonreflecting acoustic wave equation, and uses the energy of transmitted waves to scale the gradient by eliminating the effect of geometric diffusion and non-uniformity illumination on gradient. This method does not require computing and storing the Hessian matrix and its inverse, meanwhile it considerably raises the inversion accuracy for high-velocity body and the deep strata below.

\section{Theory}

Tarantola (1984) derived the gradient that can be directly applied to the time-domain FWI from the Born approximation. When density is a constant, the gradient can be calculated by

$$
\gamma(\boldsymbol{x})=\frac{2}{c(\boldsymbol{x})^{3}} \sum_{s, r} \int \dot{\psi}\left(\boldsymbol{x}, t, \boldsymbol{x}_{\boldsymbol{r}}\right) \dot{p}\left(\boldsymbol{x}, t, \boldsymbol{x}_{s}\right) d t
$$

where $\boldsymbol{x}$ denotes the spatial position of the model; $c(\boldsymbol{x})$ is the velocity at point $\boldsymbol{x} ; p\left(\boldsymbol{x}, t, \boldsymbol{x}_{s}\right)$ stands for the forward time wavefield with the source wavelet at $\boldsymbol{x}_{s} ;\left(\boldsymbol{x}, t, \boldsymbol{x}_{r}\right)$ represents the reverse time wavefield with the virtual source at $\boldsymbol{x}_{r}$; the superscript "." signifies the first-order time derivative.

The gradient calculated by Eq. (1) is influenced by geometric diffusion and non-uniformity illumination in forward time and reverse time wavefields. Without preconditioning, the velocity updates in the middle-shallow strata will dominate the inversion process, thereby resulting in slower convergence and lower inversion accuracy in the deep strata and even the whole region. Zhang et al (2011) put forward a gradient preconditioning method based on seismic wave energy. In their studies, the energy values of forward time and reverse time wavefields are defined as

$$
\begin{aligned}
& W_{s}(\boldsymbol{x})=\sum_{(s, t)} p^{2}\left(\boldsymbol{x}, t, \boldsymbol{x}_{s}\right) \\
& W_{r}(\boldsymbol{x})=\sum_{(s, r, t)} \phi^{2}\left(\boldsymbol{x}, t, \boldsymbol{x}_{\boldsymbol{r}}\right)
\end{aligned}
$$

where $p\left(\boldsymbol{x}, t, \boldsymbol{x}_{s}\right)$ denotes the forward time wavefield from the seismic source, which takes the source wavelet as a disturbance and can be obtained from the forward modelling of acoustic wave equation; $\phi\left(x, t, \boldsymbol{x}_{r}\right)$ represents 


\section{Time-domain FWI using the GPTWE}

the reverse time wavefield at the receiver point, which takes the simulated record $u\left(\boldsymbol{x}_{r}, \boldsymbol{x}_{s}\right)$ as a disturbance and can be obtained from the reverse time extrapolation of acoustic wave equation. $\gamma_{p}$ is the gradient after preconditioning and described (Plessix et al, 2004; Zhang et al, 2012) as follow:

$$
\gamma_{P}(\boldsymbol{x})=\frac{\gamma(\boldsymbol{x})}{\sqrt{W_{s}(\boldsymbol{x}) W_{r}(\boldsymbol{x})}}
$$

In essence, the gradient preconditioning method based on seismic wave energy is using the energy of seismic waves to scale the gradient by eliminating the effect of geometric diffusion and non-uniformity illumination. However, as seismic wavefield is divided into "transmitted wavefield" and "reflected wavefield", it is theoretically more accurate "approximated transmitted wavefield" simulated from the nonreflecting acoustic wave equation.

The nonreflecting acoustic wave equation can provide arough description of the transmitted wavefield. The equation is illustrated as follow (Baysal et al, 1984):

$$
\frac{\partial^{2} P}{\partial t^{2}}=v\left[\frac{\partial}{\partial x}\left(v \frac{\partial P}{\partial x}\right)+\frac{\partial}{\partial z}\left(v \frac{\partial P}{\partial z}\right)\right]
$$

where $P$ is the sound pressure and $v$ is the wave velocity. Each iteration of the time-domain FWI using the gradient preconditioning based on transmitted wave energy is accomplished following these steps. Firstly, the gradient direction is calculated from the extrapolation of forward time and reverse time wavefields by acoustic wave equation, which is the same as traditional FWI. Secondly,

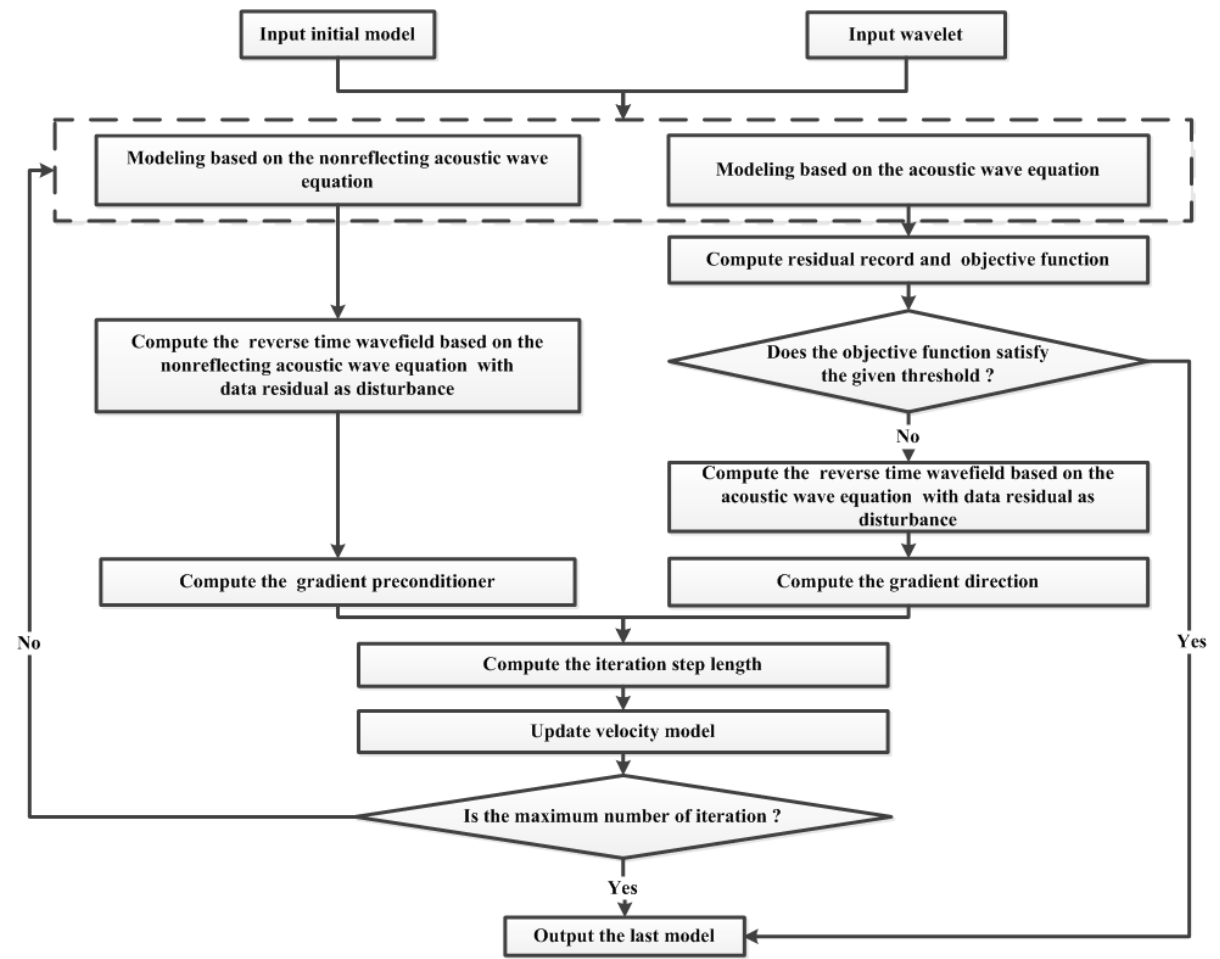

Figure 1 Flow chart of the time-domain FWI using gradient precondition based on the energy of transmission waves

to characterize the geometric diffusion and non-uniformity illumination of the gradient with the information of "transmitted wavefield". When using the gradient preconditioning method based on seismic wave energy, the wavefield simulated by the acoustic wave equation contains plenty of reflected waves apart from transmitted waves, and the accuracy of this method will be greatly influenced by the strong reflected wave energy when strong reflectors exist in the velocity model. Therefore, we put forward a gradient preconditioning method based on the transmitted wave energy, which scales the gradient with the based on the nonreflecting acoustic wave equation, acquire the "approximated transmitted wavefield" that can better describe the geometric diffusion and illumination distribution of seismic waves by simulation and used it in the gradient precondition afterwards. At last, the processed gradient is used for step length and model updating (see Figure 1 for details).

As seen from Figure 1, the time-domain FWI using the gradient preconditioning based on transmitted wave energy requires two more extrapolations of wavefield in each iteration than the traditional methods, thereby consuming more for computation. This study adopts GPU platform 


\section{Time-domain FWI using the GPTWE}

and encoding technique to improve the computational efficiency of the modified time-domain FWI. But specific procedures will not be elaborated here.

\section{Examples}

The Marmousi model (see Figure 2) is $9.2 \mathrm{~km}$ long and $3 \mathrm{~km}$ deep, with $10 \mathrm{~m}$ long grid intervals horizontally and vertically. The geometry is designed for fixed receivers and mobile shots. There are 461 shots in total, with 20 -meter width interval and 20-meter depth interval.

The initial velocity model is shown in Figure 3. The inversion test adopted 80 iterations with five frequencies for each (high cutoff frequencies: $5 \mathrm{~Hz}, 8 \mathrm{~Hz}, 15 \mathrm{~Hz}, 25 \mathrm{~Hz}$, $37 \mathrm{~Hz})$. Results of the inversion using the gradient preconditioning method based on the seismic wave energy (GPWE) and on the transmitted wave energy (GPTWE) are shown in Figure 4 and Figure 5 respectively.

Figure 6 illustrates the inversion velocity curves at 3,080m and $7,550 \mathrm{~m}$ correspondingly to the results of the inversion with GPWE and GPTWE (the black, blue, green and red lines indicate the actual velocity, the initial velocity, the velocity of the inversion using GPWE, and the velocity of the inversion using GPTWE respectively).

It can be found by comparison that the inversion of deep high-velocity model and its underlying strata using GPTWE is more accurate than that using GPWE, and the difference of velocity inversion reach about $200 \mathrm{~m} / \mathrm{s}$ (indicated by the red circle in Figure 6).

To further quantify the inversion accuracy, we adopt the factor defined by Ben-Hadj-Ali et al (2011) to estimate the error between inversion result and actual model. The estimated factor is expressed as follow:

$$
E_{\text {res }}=\frac{\left\|m_{\text {result }}-m_{\text {real }}\right\|^{2}}{\left\|m_{\text {real }}\right\|^{2}} \times 100 \%
$$

where $m_{\text {result }}$ denotes the final inversion model and $m_{\text {real }}$ denotes the actual model. The $E_{\text {res }}$ values of the inversion using GPWE and GPTWE are $1.12 \%$ and $1.05 \%$ respectively. The $E_{\text {res }}$ value of the inversion using GPTWE was lower than that using GPWE. Therefore, it can verify that the time-domain FWI using GPTWE boasts higher overall accuracy than that using GPWE.

\section{Conclusion}

We propose the gradient preconditioning approach based on the transmitted wave energy. It preconditions the gradient using the energy of the "approximated transmitted wavefiled" simulated by the nonreflecting acoustic wave equation. The approach avoids computing and storing highdimensional matrices in those algorithms based on Hessian matrices and effectively eliminates the influence of geometric diffusion and non-uniform illumination on gradient. The time-domain FWI using the gradient preconditioning approach performs well even for complex models. In addition, it can achieve higher accuracy for deep high-velocity models and their underlying strata when compared with the method based on seismic wave energy. In this study, the "approximated transmitted wavefield" simulated by the nonreflecting acoustic wave equation fails to fully suppress the reflection of wide-angle incident waves between layers. Therefore, future researches will focus on improving the simulation precision of transmitted seismic waves for more accurate gradient preconditioning operator and promoting its application in real inversions.

\section{Acknowledgments}

This work was supported by the National Natural Science Foundation of China (41574105).

\section{References}

Baysal, E., Kosloff, D. D., and Sherwood, 1984, A twoway nonreflecting wave equation, Geophysics, 49(2), 132141.

Ben-Hadj-Ali, H., Operto S., and Vineux J., 2011, An efficient frequency-domain full waveform inversion method using simultaneous encoded sources, Geophysics, 76(4), R109-R124.

Brossier R., Operto S., and Virieux J., 2009, Seismic Imaging of complex onshore structures by $2 \mathrm{D}$ elastic frequency-domain full-waveform inversion, Geophysics, 74(6), WCC105-WCC118.

Plessix R., Mudler W., 2004, Frequency-domain finitedifference amplitude-preserving migration, Geophys. J. Int., 157(3), 975-987

Pratt G., Shin C., 1998, Gauss-Newton and full Newton methods in frequency-space seismic waveform inversion, Geophys. J. Int., 133(2): 341-362.

Shin C., Yoon K. G., Marfurt K J, et al. 2001. Efficiemt calculation of a partial-derivative wavefield using reciprocity for seismic imaging and inversion, Geophysics, 66(6):1856-1863.

Tarantola A., 1984, Inversion of seismic reflection data in the acoustic approximation, Geophysics, 49(8), 1259-1266.

Wang Y., Rao Y., 2009, Reflection seismic waveform tomography, Journal of Geophysical Research, 114(B3), B03304. 


\section{Time-domain FWI using the GPTWE}

Zhang Zhigang, Lin Youzuo and Huang Lianjie, 2011, Full-waveform inversion in the time domain with an energy-weighted gradient, 81st Annual International Meeting, SEG, Expanded Abstracts, 2772-2776.

Zhang Zhigang, Huang Lianjie and Lin Youzuo. 2012. A

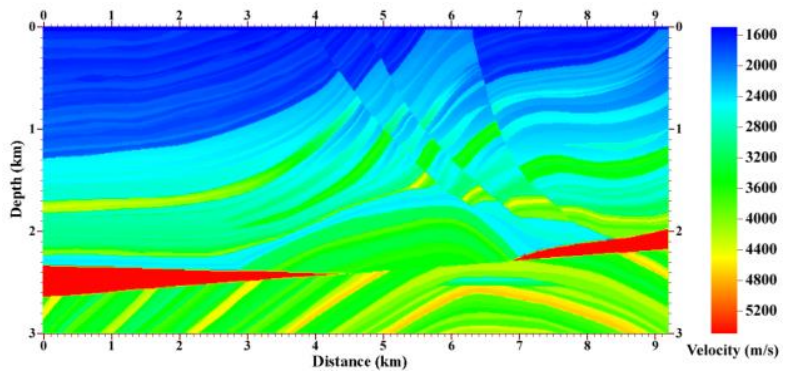

Figure 2 The Marmousi model

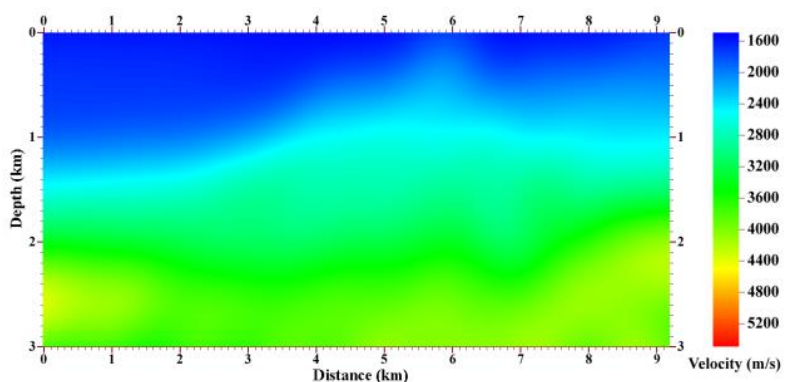

Figure 3 The initial velocity model

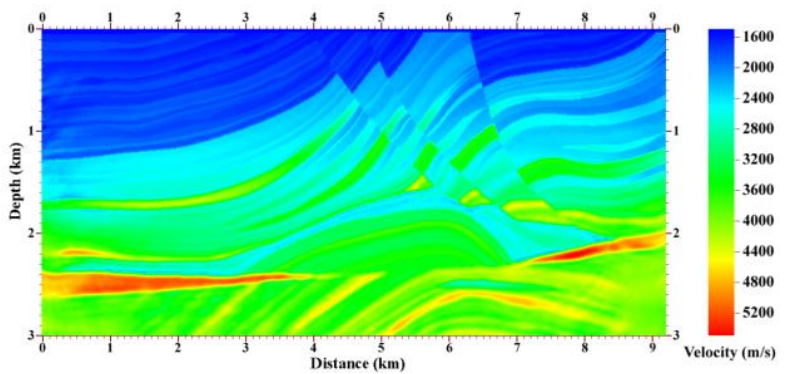

Figure 4 The result of the inversion using GPWE

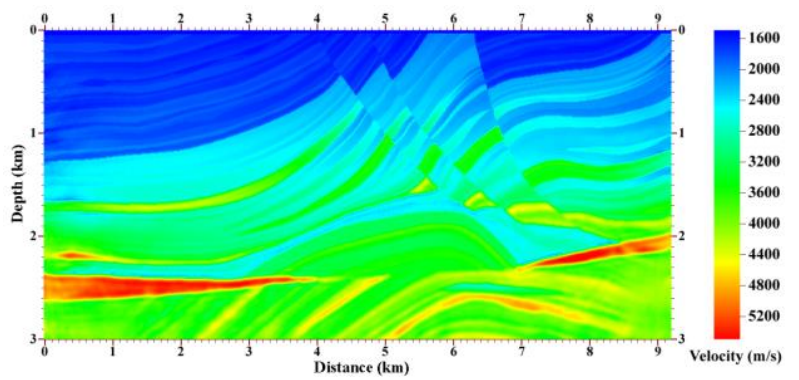

Figure 5 The result of the inversion using GPTWE wave-energy-based precondition approach to fullwaveform inversion in the time domain, 82nd Annual International Meeting, SEG, Expanded Abstracts, 1-5.
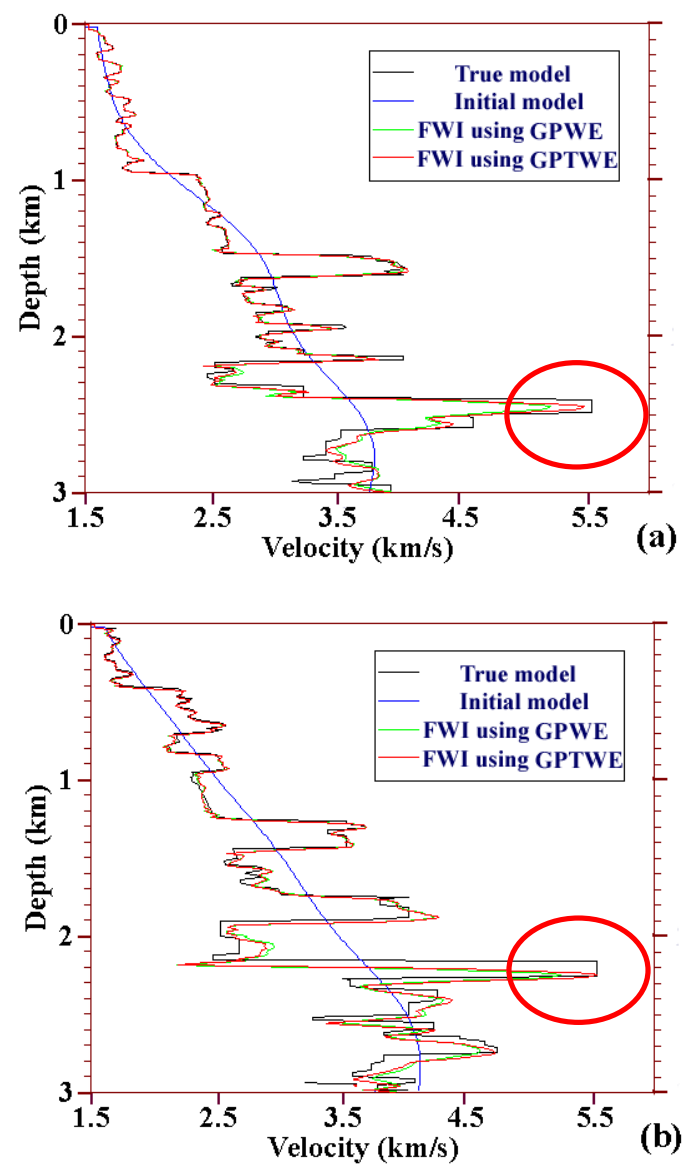

Figure 6 The velocity curves

((a) is the velocity curves at $\mathrm{x}=3080 \mathrm{~m}$; (b) is the velocity curves at $\mathrm{x}=7550 \mathrm{~m}$ ) 